Almost one-third $(228,000)$ of these engineers and scientists were engaged in research and development, and two-thirds of these were employed in the aircraft, electrical equipment, machinery or chemical industries. Nearly three out of every five physicists, but fewer than one out of three of the engineers, mathematicians and biological scientists and two out of every five chemists were engaged in research and development. Nearly 600,000 technicians worked with scientists and engineers in all activities, and of these, 160,000 were engaged in research and development, the largest numbers in the electrical equipment, aireraft, machinery, chemical and fabricated metal parts and ordnance industries. During 1954-57 employment of engineers rose by 27 per cent, of chemists by 16 per cent and of biological scientists and physicists by about 60 per cent. In these three years 70,000 first degrees in engineering were awarded by American engineering ; the proportinn of engineers and scientists employed in research and develop. ment rose from 28 to 31 per cent, and employment in such activities rose by. more than 45 per cent, whereas in the same period research and development costs rose from 3,700 million to 6,400 million dollars.

\title{
CHEMOTHERAPY OF PARASITIC DISEASES
}

A

MEETING of the Parasitology Group of the Institute of Biology was held at the Institute's rooms in London on October 24, when two speakers were invited to read papers on the chemotherapy of parasitic diseases.

Dr. Ann Bishop (Molteno Institute, Cambridge) described some aspects of resistance to drugs in parasitic Protozoa. The fact that parasitic organisms can become resistant to drugs in the course of the therapeutic treatment of the host was discovered in Ehrlich's laboratory, early in the present century, by Franke and Röhl while studying the effect of parafuchsin upon Trypanosoma brucei in mice. Trypanosomes have since been made resistant to a wide variety of chemical compounds.

The fundamental problems in the study of resist. ance to drugs are the nature of the changes which organisms undergo in becoming resistant and the means by which these changes are brought about. In trypanosomes, resistance to drugs may be associated with changes in permeability and with changes in the enzymes present in the cells. Drug resistance in these organisms may be a very stable character and persists after the transmission of the parasite through the insect vector. The spontaneous development of resistance, in the absence of the drug, has been described both in trypanosomes and in Toxoplasma gondii.

Malaria parasites also can be made resistant to certain compounds, including proguanil and pyrimethamine, which are used in the treatment of the disease. Resistance persists after transmission of the parasite through the insect vector, in which, unlike trypanosomes, the malaria parasite undergoes a sexual cycle of development.

A study has been made of the development of resistance to metachloridine (2-metanilamido-5chloropyrimidine) in pure lines of Plasmodium gallinaceum in chicks. No difference in the rate of development of resistance was observed in these lines. The rate of development of resistance was not related to the size of the dose of drug, but was affected by the size of the inocula producing the infections oxposed to the action of the drug. The suddenness with which resistance appeared in some of the strains studied, and its stability in the absence of the drug and after the sexual development of the parasite, suggested that it arose by mutation, the mutation being of a low frequency.

Dr. O. D. Standen (Wellcome Laboratories of Tropical Medicine, London) outlined an approach to the experimental chemotherapy of helminthiases. The diseases of man and animals caused by the presence of helminth parasites are particularly important in the tropies, and are often responsible for the low level of efficiency and uneconomic production of stock animals in rural areas. The eventual control of these helminthiases will depend on a fuller knowledge of their epidemiology, but also presents problems which can only be resolved by the use of drugs.

The number of even moderately efficient anthelmintics in use at the present time is small, and most are toxic to the host. There is thus an urgent need for drugs which could be used in mass treatments of man and animals.

The discovery of new drugs requires the close collaboration of biologist and chemist. Two methods of approach may be used. An empirical method is one whereby large numbers of compounds of unknown activity are tested, in order to determine their effects on the parasite. The selective approach is one in which chemicals of known anthelmintic potency are modified in order to improve their action, and this method may be used with substances discovered by the empirical method. The ultimate aim is to discover the relation between the molecular structure of the drug and its anthelmintic activity and toxicity to the host.

The selection of helminths to be cultured in laboratory animals is of great importance. The number which can be cultured in this way is small, and of these the species pathogenic to man and animals are few. It is thus necessary to test drugs using helminths which are the natural parasites of laboratory animals. The successful maintenance of a parasite in all the stages of its life-history in laboratory animals often requires a long period of fundamental research before a sufficient supply of material is assured for routine investigations, for which an abundance of material is required since hundreds or even thousands of tests may be necessary before an effective drug is discovered.

The use of a multiple helminth screen, employing a wide range of species living in different habitats, yields interesting results. Thus, basic work with Aspicularis tetraptera in the mouse has contributed materially to the development of piperazine for the treatment of ascariasis and oxyuriasis. More recent chemotherapeutic tests, against Nippostrongylus muris in the young rat, provided the basis for the development of salts of bephenium that are efficient in the treatment of nematodiriasis in sheep and ancylostomiasis in man and dogs.

The experimental chemotherapy of helminthiases necessarily demands many species of helminths in all 
stages of their development and this offers an unparalleled opportunity for concurrent research into the structure and life-cycles of the parasites, into their biochemistry and host-parasite relationships and into the mode of drug action. While such studies may be an end in themselves, they also provide the only basis for a rational approach to chemotherapy.

In opening the discussion, Dr. H. O. J. Collier (Department of Pharmacological Research, Parke, Davis and Co., Ltd., Hounslow) said that the history of practical chemotherapy began with the treatment of parasitic diseases. Experimental chemotherapy also started in this field. In 1891 Ehrlich and Guttmann (Berl. Klin. Wsch., 28 (9), 953; 1891) showed that methylene blue is a fairly potent antimalarial agent. Choosing this dye because it stained the malaria parasite within red corpuscles and because it was of low toxicity to man, Ehrlich, with Guttman's help, treated with it two sufferers from malaria and achieved immediste success. Later, Ehrlich had to test hundreds of compounds to achieve ' 606 ' and did not screen at random but approached his objective by steps, basing each new compound on results obtained with previous ones.

Work described in the preceding papers follows on the experimental chemotherapy started by Ehrlich. Each speaker was mainly concerned with one major problem, well known to Ehrlich and his colleagues; Dr. Bishop with resistance to drugs, and Dr. Standen with specificity of interaction between chemical and biological species.

Some of the ways in which drug resistance can develop were described. Compared with a sensitive organism, a resistant form may absorb a drug less, excrete it faster, destroy it more effectively, produce more of the drug's antagonist or possess a route of biosynthesis alternative to that blocked by the drug. These differences may depend on modifications of enzyme systems; an understanding of these would lead to a better understanding of drug resistance.
Problems of specificity are at the heart of chemotherapy, because if drugs were not more toxic to one species than to another, selective poisoning would be impossible. The differences in responses of various species to the same compound provide difficulties, however, because related species cannot be relied upon to respond in the same way to a new substance, and the second paper explained how the experimental chemotherapist deals with this dilemma.

Another aspect of the use of chemotherapeutic agents was mentioned by Dr. S. Kendall, in reference to coccidiosis of fowls. Drugs are added to feed in order to determine whether they are toxic to the parasite or whether resistance is developed. Results, so far, show these drugs to have a coccidiostatic action, without elimination of the parasite. Dr. L. Joyner said that this posed the general problem as to whether eradication of the parasite was necessary in the case of flukes, for example, it was desirable However, in some protozoan infections, it is preferable to induce a state of premunition in the host, or one of immunity induced by stages in the life-cycle other than the pathogenic one. Dr. J. Leiper said that some anthelmintics effective over a wide range may produce curious results, some parasites being killed and others encouraged. Thus, in the use of chemotherapeutic agents the habitat of the parasite within the host may be as important as the specific action of the drug. Dr. Standen replied that the range of activity of the drug must be carefully tested. Dr. C. Hopkins pointed out that the mode of action of the drug is unknown, a situation complicated by the difficulties of cultivation of parasites in vitro. Anthelmintics can act by blocking the metabolism of the worm, not necessarily causing a complete 'knock-out' reaction, and the site of the parasite within the host might indicate specific substances required from the host by the metabolism of the parasite.

The proceedings of this meeting will not be published in full. JUNE MAGON

\title{
A PRESUMPTIVE HUMAN XXY/XX MOSAIC
}

\author{
By DR. C. E. FORD \\ Medical Research Council Radiobiological Research Unit, Atomic Energy Research Establishment, Harwell, Berks \\ AND \\ Dr. P. E. POLANI, J. H. BRIGGS and DR. P. M. F. BISHOP \\ Guy's Hospital, London Bridge, S.E.I
}

\begin{abstract}
THE discovery of 'sex chromatin' by Barr and Bertram $^{1}$ and the subsequent recognition of its regular occurrence in normal females of many mammalian species (including man) has provided a new approach to the study of human intersexual conditions, the value of which is demonstrated in the proceedings of a recent symposium ${ }^{2}$. Although the presence of sex chromatin is generally taken to be diagnostic of an $X X$ individual, this is not proved, and the unequivocal determination of the sex chromosome constitution can only come from the direct study of the chromosomes themselves.

A technique for obtaining chromosome preparations from biopsy specimens of bone marrow has recently been described in outline and used in the study of one 'chromatin-positive' case of Kline.
\end{abstract}

felter's syndrome ${ }^{3}$. (Klinefelter's syndrome is a variable clinical syndrome in somatically male individuals who are sterile, generally with azoospermia, often with gynæcomastia and endocrine defects, but essentially and always with small testes after puberty.) The few cells from this first case seen in metaphase contained chromosomes indistinguishable from those of a normal female. Recently we have been able to examine the chromosomes from two further cases of this syndrome. One, chromatinnegative, had the chromosomes of a normal male. The second, chromatin-positive, gave an unexpected result and is the subject of this article.

A marrow specimen was obtained by sternal puncture at Guy's Hospital, converted into a suspension there and immediately sent to Harwell for 